\title{
CFT driven cosmology and the DGP/CFT correspondence
}

\author{
A.O.Barvinsky ${ }^{1,2}$, C.Deffayet ${ }^{3}$ and A.Yu.Kamenshchik ${ }^{4,5}$ \\ ${ }^{1}$ Theory Department, Lebedev Physics Institute, Leninsky Prospect 53, Moscow \\ 119991, Russia \\ ${ }^{2}$ LMPT CNRS-UMR 6083, Univ. of Tours, Parc de Grandmont, 37200 Tours, \\ France \\ ${ }^{3}$ APC UMR 7164 (CNRS, Univ. Paris 7, CEA, Obs. de Paris), 12 rue Alice \\ Domon et Léonie Duquet, 75205 Paris Cedex 13, France \\ ${ }^{4}$ Dipartimento di Fisica and INFN, via Irnerio 46, 40126 Bologna, Italy \\ ${ }^{5}$ L.D.Landau Institute for Theoretical Physcis of Russian Academy of Sciences, \\ Kosygin str. 2, 119334 Moscow, Russia
}

\begin{abstract}
We present a dual 5D braneworld picture of a recently suggested model for a microcanonical description of a $4 \mathrm{D}$ cosmology driven by a conformal field theory with a large number of quantum fields. The 5D side of the duality relation is represented by a generalized brane induced gravity model in a Schwarzschild-de Sitter bulk. The values of the bulk cosmological and the induced $4 \mathrm{D}$ cosmological constants are determined by requiring the absence of conical singularity at the de Sitter horizon of the Euclidean Schwarzschild-de Sitter bulk. Those values belong to the vicinity of the upper bound of a range of admissible values for the cosmological constant. This upper bound is enforced by the 4D CFT and coincides with the natural gravitational cutoff in a theory with many quantum species. The resulting DGP/CFT duality suggests the possibility of a new type of background independent correspondence. A mechanism for inverting the sign of the effective cosmological constant is found, which might reconcile a negative value of the primordial cosmological constant compatible with supersymmetry with the one required by inflationary cosmology.
\end{abstract}

\section{Introduction}

As is widely recognized, the AdS/CFT correspondence [1] is an efficient tool for the nonperturbative analysis of various problems, providing a new insight in the underlying physical mechanisms and relating classical and quantum domains in relevant weak and strong coupling regimes. It is natural, therefore, to look for new applications where the hypothesis of this (or a similar) correspondence can work and bring new nonperturbative results. This becomes even more interesting in the context of a recently suggested relation between the cutoff in classical black hole physics and the properties of a particle model [2, 3], 
establishing the dependence of this cutoff on the number of quantum species $N$ in the theory. The AdS/CFT correspondence relates dual models in the domains of couplings separated by this cutoff and, thus, directly involves this concept.

Together with the hierarchy problem of the electroweak vs Planckian scales [2], one of the most intriguing implications of these ideas concerns problems in early and late cosmology ranging from the origin of inflationary Universe to the dark energy. In this sense, a recently suggested cosmological model driven by a conformal field theory (CFT) with a large number of conformal fields 4, 5] is a perfect tool for such a duality analysis. This model is of a particular interest, because it incorporates a microcanonical initial conditions in cosmology, capable of resolving the string landscape problem, and possibly contains a mechanism for the cosmological acceleration [5, 6].

In this paper we show that this essentially quantum four-dimensional (4D) model has a dual description in terms of a classical 5D theory incorporating a dynamically evolving 4D brane with brane induced gravity in a Schwarzschildde Sitter 5D bulk (what we will call here the generalized DGP model). The parameters of this model - its cosmological constant $\Lambda_{5}$ and the black hole mass belong to the classical domain much below the cutoff, whereas the $4 \mathrm{D}$ cosmology of [4] on the CFT side of the duality relation lies close to the upper boundary of the admissible range of values for the $4 \mathrm{D}$ cosmological constant $\Lambda_{4}$. This range was derived in 4 and suggested to be of interest for dealing with the string landscape and the cosmological constant problem. Distinctive peculiarities of this $D G P / C F T$ correspondence is that it is realized not on the AdS background, but on the Schwarzschild-de Sitter one, and does not resort to group-theoretical and, in particular, supersymmetry arguments. Moreover, the parameters of the system (that is its cosmological constant $\Lambda_{5}=2 \Lambda_{4}$, temperature and the radiation energy or the black hole mass on the $5 \mathrm{D}$ side) are not freely specifiable, but are entirely determined in terms of the Planck mass by the particle content of the model.

The organization of the paper is as follows. In Sect.2 we recapitulate the construction of the CFT driven cosmology of [4, 5], its initial conditions prescribed by the microcanonical density matrix and late time evolution incorporating the cosmological acceleration in the form of the Big Boost scenario [6]. In Sect.3 we discuss the single branch generalized DGP model with a positive $\Lambda_{5}$ in the bulk, which for the vacuum case was observed in 6 to be equivalent to the CFT cosmology. Sect. 4 presents the derivation of this equivalence in the Euclidean signature spacetime which underlies the $4 \mathrm{D}$ cosmological instantons dominating the statistical sum path integral. On the 5D side these instantons represent a dynamically evolving brane embedded into the Schwarzschild-de Sitter bulk with $\Lambda_{5}=2 \Lambda_{4}$, its black hole mass being related to the thermal radiation on the brane. Complete duality of $4 \mathrm{D}$ and 5D descriptions implies the specification of the cosmological constant and the radiation energy, which on the 4D side follows from the quantum Einstein equations and on the $5 \mathrm{D}$ side is enforced by the requirement of the absence of conical singularity in the bulk geometry. Together these conditions select from the finite range of $\Lambda_{4}$, admissible from the $4 \mathrm{D}$ viewpoint, a value close to the upper limit of this range, where the gravitating CFT 
belongs to a strong coupling regime treated within $1 / N$-expansion. This property is derived in Sect.5 for a special set of static Einstein instantons of the CFT cosmology and for the non-static solutions called garlands in [4 — the instantons giving rise to expanding inflationary models. Sect. 6 contains an attempt to generalize this DGP/CFT correspondence to the case of the background independent formulation, when the field configuration is not limited by symmetry or weak field linearization restrictions - an idea suggested in [7] on the basis of quantum information and gravity cutoff bounds. In particular, it is shown that a full nonlinear trace part of effective Einstein equations can be exactly the same on both sides of the duality relation. This turns out to be true under the assumption of a particle phenomenology motivated by the superconformal $\mathcal{N}=4$ Yang-Mills theory which underlies the $A d S_{5} \times S_{5} / S C F T$ correspondence of [1. In Sect.7 we discuss an interesting mechanism based on the conformal anomaly to reverse the sign of the primordial negative cosmological constant. This could reconcile supersymmetry with inflation. Sect. 8 contains conclusions and a brief discussion of the results.

\section{CFT driven cosmology}

The initial conditions for the CFT driven cosmology of [4, 5] were prescribed in terms of a microcanonical density matrix. They are based on the corresponding statistical sum for a spatially closed cosmology ( $S^{3}$-topology of spatial sections). It is represented by the path integral over the periodic scale factor $a(\tau)$ and lapse function $N(\tau)$ of the minisuperspace metric

$$
d s^{2}=N^{2}(\tau) d \tau^{2}+a^{2}(\tau) d^{2} \Omega^{(3)}
$$

on the toroidal spacetime of $S^{1} \times S^{3}$ topology

$$
e^{-\Gamma}=\int_{\text {periodic }} D[a, N] e^{-\Gamma_{E}[a, N]} .
$$

Here $\Gamma_{E}[a, N]$ is the Euclidean effective action of all inhomogeneous "matter" fields on the minisuperspace background (11) including also the Einstein action with with the renormalized gravitational $G$ and cosmological $\Lambda$ constants. This action incorporates the integration over all degrees of freedom of the system not contained in its minisuperspace sector.

Under the assumption that the system is dominated by free matter fields conformally coupled to gravity this action is exactly calculable by the conformal transformation converting (1) into the static Einstein metric with $a=$ const [4]. 
In units of the Planck mass $m_{P}=(3 \pi / 4 G)^{1 / 2}$ the action reads

$$
\begin{gathered}
\Gamma_{E}[a, N]=m_{P}^{2} \int d \tau N\left\{-a a^{\prime 2}-a+\frac{\Lambda}{3} a^{3}\right. \\
\left.+B\left(\frac{a^{\prime 2}}{a}-\frac{a^{\prime 4}}{6 a}\right)+\frac{B}{2 a}\right\}+F(\eta), \\
F(\eta)= \pm \sum_{\omega} \ln \left(1 \mp e^{-\omega \eta}\right), \quad \eta=\int \frac{d \tau N}{a},
\end{gathered}
$$

where $a^{\prime} \equiv d a / N d \tau$. The first three terms in curly brackets of (3) represent the Einstein action with a primordial (but renormalized by quantum corrections) cosmological constant

$$
\Lambda \equiv 3 H^{2}
$$

( $H$ is the corresponding Hubble constant), the $B$-terms correspond to the contribution of the conformal anomaly 8 and the contribution of the vacuum (Casimir) energy $(B / 2 a)$ of conformal fields on a static Einstein spacetime. $F(\eta)$ is the free energy of these fields - a typical boson or fermion sum over field oscillators with energies $\omega$ on a unit 3-sphere, $\eta$ playing the role of the inverse temperature - an overall circumference of the toroidal instanton measured in units of the conformal time. The constant $B$,

$$
B=\frac{3 \beta}{4 m_{P}^{2}}=\frac{\beta G}{\pi},
$$

is determined by the coefficient $\beta$ of the topological Gauss-Bonnet invariant $E=R_{\mu \nu \alpha \gamma}^{2}-4 R_{\mu \nu}^{2}+R^{2}$ in the overall conformal anomaly of quantum fields

$$
g_{\mu \nu} \frac{\delta \Gamma_{A}}{\delta g_{\mu \nu}}=\frac{1}{4(4 \pi)^{2}} g^{1 / 2}\left(\alpha \square R+\beta E+\gamma C_{\mu \nu \alpha \beta}^{2}\right)
$$

Here $\Gamma_{A}$ is the CFT action in the external gravitational field generating the conformal anomaly and $C_{\mu \nu \alpha \beta}^{2}$ is the Weyl tensor squared term. For a model with $N_{0}$ scalars, $N_{1 / 2}$ Weyl spinors and $N_{1}$ gauge vector fields it reads [12]

$$
\beta=\frac{1}{360}\left(2 N_{0}+11 N_{1 / 2}+124 N_{1}\right)
$$

The status of other coefficients of (7) is as follows. The coefficient $\gamma$ does not contribute to (3) because the Weyl tensor vanishes for any FRW metric. A nonvanishing $\alpha$ induces higher derivative terms $\sim \alpha\left(a^{\prime \prime}\right)^{2}$ in the action and, therefore, adds one extra degree of freedom to the minisuperspace sector of $a$ and $N$ which generically leads to instabilities 1 . But $\alpha$ can be renormalized to zero by

\footnotetext{
${ }^{1}$ In Einstein theory this sector does not contain physical degrees of freedom at all, which solves the problem of the formal ghost nature of $a$ in the Einstein Lagrangian. Addition of higher derivative term for $a$ does not necessarily lead to a ghost - the additional degree of freedom can have a good sign of the kinetic term as it happens in $f(R)$-theories, but still leads to the instabilities discovered in [9].
} 
adding a finite local counterterm $\sim R^{2}$ admissible by the renormalization theory. Such a number of degrees of freedom preserving renormalization was assumed in [4] to keep the theory consistent both at the classical and quantum levels. It is important that this finite renormalization changes the value of the Casimir energy of conformal fields in closed Einstein cosmology in such a way that for all spins this energy is universally expressed in terms of the same conformal anomaly coefficient $B$ (corresponding to the $B / 2 a$ term in (3i) ) [13, 4] 2 .

The path integral (2) is dominated by saddle points - solutions of the equation $\delta \Gamma_{E} / \delta N(\tau)=0$ which reads as

$$
-\frac{a^{\prime 2}}{a^{2}}+\frac{1}{a^{2}}-\frac{B}{2}\left(-\frac{a^{\prime 2}}{a^{2}}+\frac{1}{a^{2}}\right)^{2}=\frac{\Lambda}{3}+\frac{\mathcal{C}}{a^{4}}
$$

with $\mathcal{C}$ given by

$$
\mathcal{C}=\frac{1}{m_{P}^{2}} \frac{d F(\eta)}{d \eta}=\frac{1}{m_{P}^{2}} \sum_{\omega} \frac{\omega}{e^{\omega \eta} \mp 1}, \quad \eta=\oint \frac{d \tau}{a} \equiv 2 k \int_{\tau_{-}}^{\tau_{+}} \frac{d \tau}{a} .
$$

This Euclidean Friedmann equation is modified by the anomalous $B$-term and the radiation term $\mathcal{C} / a^{4}$. The constant $\mathcal{C}$ sets the amount of radiation and determines the energy of the gas of thermally excited particles with the inverse temperature $\eta$ - the instanton period in units of the conformal time. The latter is given in (10) by the integral over the full period of $\tau$ or the $2 k$-fold integral between the two neighboring turning points of the scale factor history $a(\tau)$, $\dot{a}\left(\tau_{ \pm}\right)=0$. This $k$-fold nature implies that in the periodic solution the scale factor oscillates $k$ times between its maximum and minimum values $a_{ \pm}=a\left(\tau_{ \pm}\right)$, $a_{-} \leq a(\tau) \leq a_{+}$,

$$
a_{ \pm}^{2}=\frac{1}{2 H^{2}}\left(1 \pm \sqrt{1-2 B H^{2}-4 H^{2} \mathcal{C}}\right) .
$$

Thus, the period of the solutions is determined as a function of $G$ and $\Lambda$ from the second of Eqs.(10) and is not freely specifiable. This is the artifact of a microcanonical ensemble (see [5]) with only two freely specifiable dimensional parameters - the renormalized gravitational and renormalized cosmological constants.

As shown in [4, such solutions represent $S^{3} \times S^{1}$ garland-type instantons which exist only in the limited range of the cosmological constant $\Lambda=3 H^{2}$

$$
0<H_{\min }^{2}<H^{2}<\frac{\pi}{2 \beta G}=\frac{1}{2 B} .
$$

In this range they form an infinite $k=0,1,2, \ldots$ sequence of one-parameter families depicted in Fig 1 in the two-dimensional plane of the Hubble constant $H^{2}$ and the amount of radiation constant $C=\mathcal{C}+B / 2$ (including together with

\footnotetext{
${ }^{2}$ This universality property follows from the fact that in a conformally flat spacetime the case of a decompactified universe $R \times S^{3}$ - the Casimir energy is entirely determined by the conformal anomaly coefficients [13. We thank P.Mazur and E.Mottola for the discussion of this point.
} 
the energy of the radiation gas $\mathcal{C}$, see (10), also the Casimir energy constant $B / 2$.) These families interpolate between the two boundaries of a curvilinear triangle of the instanton domain in the $\left(H^{2}, C\right)$-plane - the lower straight line boundary $C=B-B^{2} H^{2}$ and the upper hyperbolic boundary $C=1 / 4 H^{2}$. Their sequence at $k \rightarrow \infty$ accumulates at the corner of this triangle - the upper bound of the range (12) $H^{2}=1 / 2 B$.

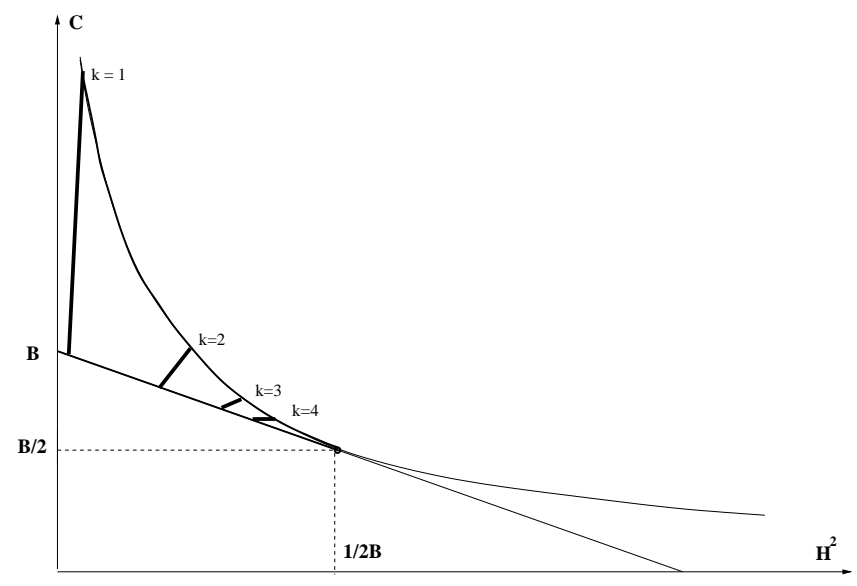

Figure 1: The instanton domain in the $\left(H^{2}, C\right)$-plane with four garland families $k=$ $1,2,3,4$. $C=\mathcal{C}+B / 2$ is the amount of radiation constant including the contribution of the Casimir energy.

This bound represents a new quantum gravity scale which tends to infinity when one switches the quantum effects off, $\beta \rightarrow 0$. The lower bound $H_{\min }^{2}-$ the lowest point of $k=1$ family — can be obtained numerically for any field content of the model. For a large number of conformal fields $N$, and therefore a large $\beta \sim N$, the lower bound is of the order $H_{\min }^{2} \sim 1 / \beta G$. Thus the restriction (12) suggests a kind of $1 / N$ solution of the cosmological constant problem, because specifying a sufficiently high number of conformal fields one can achieve a primordial value of $\Lambda$ well below the Planck scale where the effective theory applies, but high enough to generate a sufficiently long inflationary stage. Also this restriction can be potentially considered as a selection criterion for the landscape of string vacua [4, 5.

The solutions of the system (91)-(10) include the vacuum Hartle-Hawking instantons of 10 (see their dual braneworld description in 11]) with no radiation $\mathcal{C}=0$. They represent the Euclidean de Sitter spacetime with the effective Hubble factor

$$
H_{\mathrm{eff}}^{2}=\frac{1-\sqrt{1-2 B H^{2}}}{B},
$$

corresponding to the degenerate case when a torus $S^{3} \times S^{1}$ gets ripped at the vanishing value of the scale factor $a_{-}=0$ and topologically becomes a 4 -sphere $S^{4}$ - a purely vacuum contribution to the statistical sum. The vacuum nature 
of these instantons follows from the fact that their conformal time period in (10) is divergent at $\tau_{-}$in view of $a_{-}=0$ and generates zero effective temperature $\sim 1 / \eta$ with $F(\eta)=0$. Such solutions exist for all $H^{2} \leq 1 / 2 B$ (the horizontal segment at $C \equiv \mathcal{C}+B / 2=B / 2$ in Fig 1 , but they are ruled out in the statistical sum by their infinite positive effective action. This property is due to the contribution of the conformal anomaly (cf. 1/a-dependence in the kinetic $B$-terms of the effective action (3) 3 . Hence the tree-level predictions of the theory with a negative Euclidean action are drastically changed by the effect of the conformal anomaly.

The solutions of (9)-(10) also include the exactly static instantons with $a_{-}=$ $a_{+}=1 / H \sqrt{2}$ which occupy the full upper hyperbolic boundary of the instanton domain in Fig 1. They exist for all $H^{2}$ from the range (12), but they are not interesting from the viewpoint of the early Universe, because they stay static and do not give rise to expanding cosmological models. As they serve as a simple working tool for the DGP/CFT selection rules, they will be considered in much detail in Sect.5 below.

The quadratic equation (9) can be solved for the Euclidean Hubble factor squared $\left(1-a^{\prime 2}\right) / a^{2}$. For periodic instantons of the above type only one root is admissible 4 , which gives

$$
-\frac{a^{\prime 2}}{a^{2}}+\frac{1}{a^{2}}=\frac{1}{B}\left\{1-\sqrt{1-2 B\left(\frac{\Lambda}{3}+\frac{\mathcal{C}}{a^{4}}\right)}\right\} .
$$

This equation obviously recovers the Einstein theory in the limit of a small $B$ and low matter density $\varepsilon \equiv(3 / 8 \pi G)\left(\Lambda / 3+\mathcal{C} / a^{4}\right), G B \varepsilon \ll 1$, because its right hand side takes in this limit a usual GR form $8 \pi G \varepsilon / 3$ independent of $B$. Also it shows that the Casimir energy that was initially present in the effective action (3), the term $a^{3} \times B / 2 a^{4}$, does not weigh. In a static Einstein Universe this energy component has the same equation of state as radiation [14, but in contrast to the real radiation with the Planck (or Bose-Fermi) spectrum (10) it does not enter the right hand side of (14). This degravitation is a result of the number of degrees of freedom preserving renormalization mentioned above.

The gravitational instantons of the above type serve as a source of initial conditions for the cosmological evolution in the physical spacetime. Lorentzian signature Universes nucleate from the minimal surface of these instantons at $\tau_{+}$ - the point of the maximal expansion of their Euclidean solutions. The latter when analytically continued to the complex plane by the rule $\tau=\tau_{+}+i t$ give the evolution in real Lorentzian time $t$. With $\dot{a} \equiv d a / d t=i a^{\prime}$ the generalized

\footnotetext{
${ }^{3}$ Note that on the vacuum solution of (9) $a^{\prime 2}\left(\tau_{-}\right)=1$, and the integrand of (3) tends to $+\infty$ at $\tau_{-}$with $a \rightarrow 0$. In its turn, the absence of a conical singularity at the cap of the $S^{4}$ instanton follows from a special UV renormalization which eradicates higher-order derivatives from the effective Friedmann equation and simultaneously renders a particular value of the Casimir energy $B / 2$ [4].

${ }^{4}$ Only this sign of the square root in (14) is possible because otherwise the Euclidean solutions with $a^{\prime 2} \geq 0$ exist only outside of the domain $a_{-}<a<a_{+}$and violate the periodicity requirement imposed by the construction of the path integral for the statistical sum.
} 
Friedmann equation (14) for this evolution takes the form in the Lorentzian regime

$$
\frac{\dot{a}^{2}}{a^{2}}+\frac{1}{a^{2}}=\frac{1}{B}\left\{1-\sqrt{1-2 B\left(\frac{\Lambda}{3}+\frac{\mathcal{C}}{a^{4}}\right)}\right\} .
$$

As shown in [6], this equation first admits the stage of inflationary (quasiexponential) expansion driven by $\Lambda$ and terminating with the the usual exit scenario when $\Lambda$ is a decaying composite field (inflaton). During this stage a particle production of a conformally non-invariant matter takes over the polarization effects of conformal fields. After the thermalization this matter gives rise to an energy density $\varepsilon$ which replaces in (15) the energy density of the primordial cosmological constant and radiation, $\Lambda / 3+\mathcal{C} / a^{4} \rightarrow 8 \pi G \varepsilon / 3$. Then (15) takes the form

$$
\frac{\dot{a}^{2}}{a^{2}}+\frac{1}{a^{2}}=\frac{\pi}{\beta G}\left\{1-\sqrt{1-\frac{16 G^{2}}{3} \beta \varepsilon}\right\},
$$

where we expressed $B$ according to (6) and, due to square root non-analyticity, incorporates the so-called big boost scenario [6. In the vicinity of a vanishing square root argument the deceleration of the Universe can go over into acceleration followed by a big boost singularity at $1-16 \beta G^{2} \varepsilon / 3=0$ : at a finite value of the Hubble factor cosmological acceleration becomes infinit 5 , $\ddot{a} \rightarrow+\infty$. This branching point of Eq. (16) can be reached for a decreasing $\varepsilon$ if $\beta$ is promoted to the level of an adiabatically growing variable - the mechanism of evolving number of light Kaluza-Klein or winding modes, induced by evolving non-stabilized extra dimensions [5, 6].

\section{The generalized single-branch DGP cosmol- ogy}

The fact that the CFT driven cosmology of the above type has a dual braneworld description was first observed in [6]. The dual picture is given by a generalized DGP model [15] including together with 4D and 5D Einstein-Hilbert terms also a $5 \mathrm{D}$ cosmological constant, $\Lambda_{5}$, in the special case of a vacuum state with a vanishing matter density on the brane. Interestingly, this vacuum DGP cosmology exactly corresponds to the model of [4] with the 4D cosmological constant $\Lambda$ simulated by the $5 \mathrm{D}$ cosmological constant $\Lambda_{5}$.

Indeed, in this model (provided one neglects the bulk curvature), gravity interpolates between a $4 \mathrm{D}$ behaviour at small distances and a $5 \mathrm{D}$ behaviour at

\footnotetext{
${ }^{5}$ This singularity should be regulated by a certain UV completion of the model or suppressed by the $R^{2}$-term in the action, disregarded above in the $1 / N$-approximation. Eradication of $R^{2}$-terms by the number of degrees of freedom preserving renormalization applies in the large CFT sector of the theory, but this does not prohibit such terms in other sectors of the full model. We thank A.A.Starobinsky for this observation.
} 
large distances, with the crossover scale between the two regimes being given by $r_{c}$,

$$
\frac{G_{5}}{2 G}=r_{c}
$$

and in the absence of stress-energy exchange between the brane and the bulk, the modified Friedmann equation takes the form [16.

$$
\frac{\dot{a}^{2}}{a^{2}}+\frac{1}{a^{2}}-r_{c}^{2}\left(\frac{\dot{a}^{2}}{a^{2}}+\frac{1}{a^{2}}-\frac{8 \pi G}{3} \rho\right)^{2}=\frac{\Lambda_{5}}{6}+\frac{\mathcal{C}}{a^{4}} .
$$

Here $\mathcal{C}$ is the integration of the bulk Einstein's equation, which corresponds to a nonvanishing Weyl tensor in the bulk (or a mass of the 5D Schwarzschild black hole) 17, 18, 19 and $\rho$ is the energy density on the brane. This equation with $\rho=0$ exactly coincides with the Lorentzian version of the effective Friedmann equation in the CFT cosmology (9) under the identifications

$$
\begin{aligned}
& B \equiv \frac{\beta G}{\pi}=2 r_{c}^{2}, \\
& \Lambda=\frac{\Lambda_{5}}{2} .
\end{aligned}
$$

These identifications imply that in the DGP limit $G \ll r_{c}^{2}$, the anomaly coefficient $\beta$ is much larger than 1 , and this looks very much like the generation of the vacuum DGP model for any value of the dark radiation $\mathcal{C} / a^{4}$ from the $\mathrm{CFT}$ cosmology with a very large $\beta \sim m_{P}^{2} r_{c}^{2} \gg 1$.

However, there are several differences. First, the CFT driven DGP model does not incorporate the self-accelerating branch [16, 20] of the conventional DGP cosmology. This corresponds to the fact that only one sign of the square root is admissible in Eq. (15) - a property dictated by the instanton initial conditions at the nucleation of the Lorentzian spacetime from the Euclidean one. So, one does not have to worry about possible instabilities associated with the self-accelerating branch [21].

Another difference concerns the way the matter energy density manifests itself in the Friedmann equation for the non-vacuum case. In the CFT model it enters the right hand side of the equation as a result of the decay of the effective $4 \mathrm{D}$ cosmological constant $\Lambda$ resulting in (16). In the conventional DGP model it appears inside the parenthesis of the left hand side of equation (18), so that the DGP Hubble factor reads as

$$
\frac{\dot{a}^{2}}{a^{2}}+\frac{1}{a^{2}}=\frac{8 \pi G}{3} \rho+\frac{1}{2 r_{c}^{2}}\left\{1-\sqrt{1-4 r_{c}^{2}\left(\frac{\Lambda_{5}}{6}+\frac{\mathcal{C}}{a^{4}}-\frac{8 \pi G}{3} \rho\right)}\right\}
$$

(note the negative sign of $\rho$ under the square root and the extra first term on the right hand side).

Due to the presence of the cosmological constant in the bulk this generalized DGP model also admits the big boost acceleration scenario [6]. Indeed, for 
positive $\Lambda_{5}$ satisfying a very weak bound

$$
\Lambda_{5}>\frac{3}{2 r_{c}^{2}}
$$

Eq.(21) has a solution for which, during the cosmological expansion with $\rho \rightarrow 0$, $\mathcal{C} / a^{4} \rightarrow 0$, the argument of the square root vanishes and the acceleration tends to infinity. For that to happen, one does not actually need a growing $r_{c}$ or $\beta$, like in the CFT driven model above (see the end of the previous section). The DGP crossover scale $r_{c}$ can be constant, and the big boost singularity will still occur provided the lower bound (22) is satisfied. For a positive $\Lambda_{5}$ below this bound, the acceleration stage is eternal with an asymptotic value of the Hubble factor given by

$$
\left.\frac{\dot{a}^{2}}{a^{2}}\right|_{a \rightarrow \infty} \rightarrow \frac{1-\sqrt{1-2 r_{c}^{2} \Lambda_{5} / 3}}{2 r_{c}^{2}}, \quad 0<\Lambda_{5} \leq \frac{3}{2 r_{c}^{2}}
$$

whereas for $\Lambda_{5} \leq 0$ the model bounces from some maximal value of a scale factor and starts recollapsing.

\section{The generalized Euclidean DGP model}

The Euclidean action of the generalized vacuum DGP model of the above type reads

$$
\begin{aligned}
S_{D G P}\left[G_{A B}(X)\right]= & -\frac{1}{16 \pi G_{5}} \int_{\mathbf{B}} d^{5} X G^{1 / 2}\left(R^{(5)}\left(G_{A B}\right)-2 \Lambda_{5}\right) \\
& -\int_{\mathbf{b}} d^{4} x g^{1 / 2}\left(\frac{1}{8 \pi G_{5}}[K]+\frac{1}{16 \pi G_{4}} R\left(g_{\mu \nu}\right)\right) .
\end{aligned}
$$

Here the $5 \mathrm{D}$ bulk $\mathbf{B}$ is $Z_{2}$ symmetric with respect to the brane $\mathbf{b}$ bearing the 4D Einstein action for the induced metric $g_{\mu \nu}(x)$ and the Gibbons-Hawking term for the bulk Einstein action for the metric $G_{A B}(X)$. The latter involves the jump of the trace of the extrinsic curvature across the brane $[K]=2 K$. Variational equations for this action include the Einstein equations in the bulk and the Israel junction conditions on the brane

$$
\begin{aligned}
& R_{A B}^{(5)}-\frac{1}{2} G_{A B} R^{(5)}=-\Lambda_{5} G_{A B}, \\
& \left(K_{\mu \nu}-K g_{\mu \nu}\right)_{\mathbf{b}}=4 \pi G_{5} S_{\mu \nu},
\end{aligned}
$$

where $S_{\mu \nu}$ is a surface stress tensor on the brane coinciding with the 4D Einstein tensor

$$
S_{\mu \nu}=-\frac{1}{8 \pi G_{4}}\left(R_{\mu \nu}-\frac{1}{2} g_{\mu \nu} R\right) .
$$


If we apply these equations to the spherically symmetric ansatz for the $5 \mathrm{D}$ metric, then we get the Schwarzschild-de Sitter solution with the Schwarzschild $R_{S}$ and de Sitter horizon $R_{d S}$ radii

$$
\begin{aligned}
& d s_{(5)}^{2}=f(R) d T^{2}+\frac{d R^{2}}{f(R)}+R^{2} d \Omega_{(3)}^{2}, \\
& f(R)=1-H^{2} R^{2}-\frac{R_{S}^{2}}{R^{2}}, \\
& H^{2}=\frac{1}{R_{d S}^{2}}=\frac{\Lambda_{5}}{6},
\end{aligned}
$$

with the spherical brane embedded into the bulk according to the equations

$$
\begin{aligned}
& R=a(\tau), \\
& T=T(\tau) .
\end{aligned}
$$

Here $\tau$ is the proper time on the brane which has the induced FRW metric (11) with the lapse $N=1$ and the scale factor $a(\tau), d s_{(4)}^{2}=d \tau^{2}+a^{2}(\tau) d \Omega_{(3)}^{2}$. This embedding implies the evolution law for the $5 \mathrm{D}$ time coordinate $T(\tau)$ $\left(T^{\prime}(\tau) \equiv d T(\tau) / d \tau\right)$

$$
T^{\prime}(\tau)=\frac{\sqrt{f(a)-a^{\prime 2}}}{f(a)}
$$

and the following expression for the outward pointing normal to the boundary of the domain $R \geq a(\tau)[19$

$$
n^{A}=\left(\frac{a^{\prime}}{f},-\sqrt{f(a)-a^{\prime 2}}, 0,0,0\right) .
$$

The nonvanishing components of the respective extrinsic curvature $K_{\mu \nu}=\nabla_{\mu} n_{\nu}$ have the form

$$
\begin{aligned}
& K_{i j}=-\frac{\sqrt{f-a^{2}}}{a} g_{i j}, \\
& K_{\tau \tau}=-\frac{1}{a^{\prime}} \frac{d}{d \tau} \sqrt{f-a^{2}},
\end{aligned}
$$

where $g_{i j}=a^{2}(\tau) \gamma_{i j}$ is a metric of the 3 -sphere of the radius $a(\tau)$.

Since

$$
\begin{aligned}
& R_{\tau \tau}^{(4)}-\frac{1}{2} g_{\tau \tau} R^{(4)}=3\left(\frac{a^{\prime 2}}{a^{2}}-\frac{1}{a^{2}}\right), \\
& R_{i j}^{(4)}-\frac{1}{2} g_{i j} R^{(4)}=-g_{i j}\left(\frac{1-a^{\prime 2}}{a^{2}}-2 \frac{a^{\prime \prime}}{a}\right)
\end{aligned}
$$

and

$$
\begin{aligned}
& K_{\tau \tau}-K g_{\tau \tau}=-g^{i j} K_{i j}=3 \frac{\sqrt{f-a^{\prime 2}}}{a} \\
& K_{i j}-K g_{i j}=\left(2 \frac{\sqrt{f-a^{\prime 2}}}{a}+\frac{1}{a^{\prime}} \frac{d}{d \tau} \sqrt{f-a^{\prime 2}}\right) g_{i j}
\end{aligned}
$$


the Israel junction conditions on the brane become [19]

$$
\begin{aligned}
& \frac{\sqrt{f-a^{\prime 2}}}{a}=r_{c}\left(\frac{1}{a^{2}}-\frac{a^{\prime 2}}{a^{2}}\right), \\
& \frac{d}{d \tau}\left(a \sqrt{f-a^{\prime 2}}+r_{c} a^{\prime 2}\right)=0,
\end{aligned}
$$

the second of them being just a time derivative of the first one.

Thus, the only independent dynamical equation of brane embedding reads as (41) which is exactly the effective Friedmann equation in the CFT driven cosmology

$$
\frac{1}{a^{2}}-\frac{a^{\prime 2}}{a^{2}}=r_{c}^{2}\left(\frac{1}{a^{2}}-\frac{a^{\prime 2}}{a^{2}}\right)^{2}+\frac{\Lambda_{5}}{6}+\frac{R_{S}^{2}}{a^{4}}
$$

with the identifications of $4 \mathrm{D}$ and $5 \mathrm{D}$ gravitational and cosmological constants (19)-(20) and the following relation between the amount of the $4 \mathrm{D}$ radiation and the black hole mass (Schwarzschild radius)

$$
\mathcal{C}=R_{S}^{2} \text {. }
$$

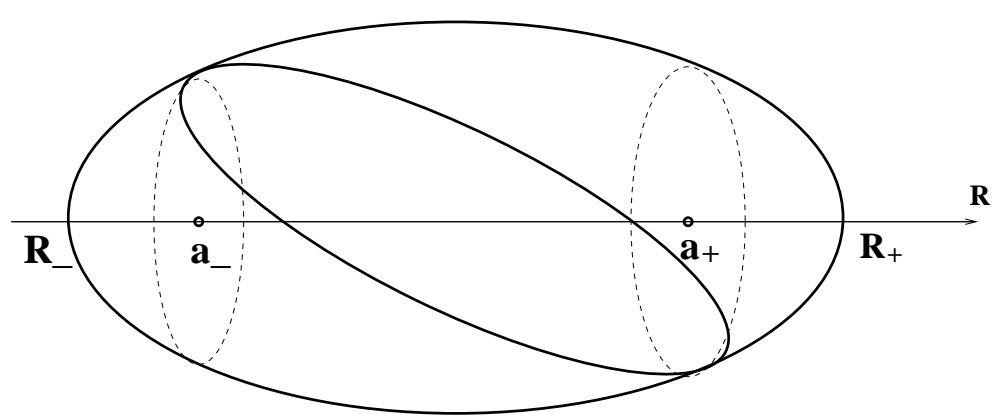

Figure 2: A part, $a_{-} \leq R \leq a_{+}$, of the 5D Schwarzschild-de Sitter Euclidean bulk (embedded in the auxiliary $6 \mathrm{D}$ spacetime) scanned by the $4 \mathrm{D}$ brane with the induced geometry of the $k=1$ garland instanton. Every point of this cigar instanton represents a 3-sphere of radius $R$.

The Euclidean signature of the Schwarzschild-de Sitter metric (28) holds for $f(R)>0$ and corresponds to a cigar-like spacetime of a finite length

$$
\begin{aligned}
& R_{-}<R<R_{+} \\
& R_{ \pm}^{2}=\frac{1}{2 H^{2}}\left(1 \pm \sqrt{1-4 H^{2} \mathcal{C}}\right)
\end{aligned}
$$

when considered from the viewpoint of the higher-dimensional embedding space (see Fig.2). Therefore, in view of (11) the 4D CFT cosmology instantons with the scale factor oscillating between $a_{ \pm}$scan only a part of this cigar 5D instanton without reaching its poles at $R_{ \pm}$depicted in Fig 2 ,

$$
R_{-}<a_{-} \leq a<a_{+} \leq R_{+}
$$


Simultaneously with $R=a(\tau)$ the Euclidean embedding time $T$ evolves in $\tau$ according to (33). This Killing coordinate of the 5D metric plays the role of the angular coordinate which should be identified with the period prescribed by the periodicity of the embedded brane

$$
\Delta T=\oint d \tau \frac{\sqrt{f(a)-a^{\prime 2}}}{f(a)}=2 k \int_{a_{-}}^{a_{+}} d a \frac{\sqrt{f(a)-a^{\prime 2}}}{\dot{a} f(a)},
$$

where $k$ in the second equality arises for the $\mathrm{k}$-fold garland case with $k$ oscillations of $a(\tau)$ between its minimal and maximal values.

On the other hand, the period of $T$ dictated by the absence of a conical singularity at $R_{ \pm}$equals $\left(f^{\prime}(R) \equiv d f(R) / d R\right)$

$$
\Delta T_{ \pm}=\frac{4 \pi}{\left|f^{\prime}\left(R_{ \pm}\right)\right|}=\frac{\pi \sqrt{2}}{H}\left(\frac{1 \pm \sqrt{1-4 R_{S}^{2} H^{2}}}{1-4 R_{S}^{2} H^{2}}\right)^{1 / 2} .
$$

As is known 23, 24 these two periods for the Schwarzschild-de Sitter solution can be identified only for the degenerate case of coinciding sizes of Schwarzschild and de Sitter horizons $R_{-}=R_{+}$, which is interpreted as the equilibrium of Hawking radiation flows emitted by these two horizon surfaces. In view of (47) this is impossible in our case, so that both conical singularities cannot be simultaneously eliminated. However, in the presence of the brane (with the $Z_{2}$-identification across it) only the region of SdS spacetime $a(\tau) \leq R \leq R_{+}$ remains (see Fig 3).

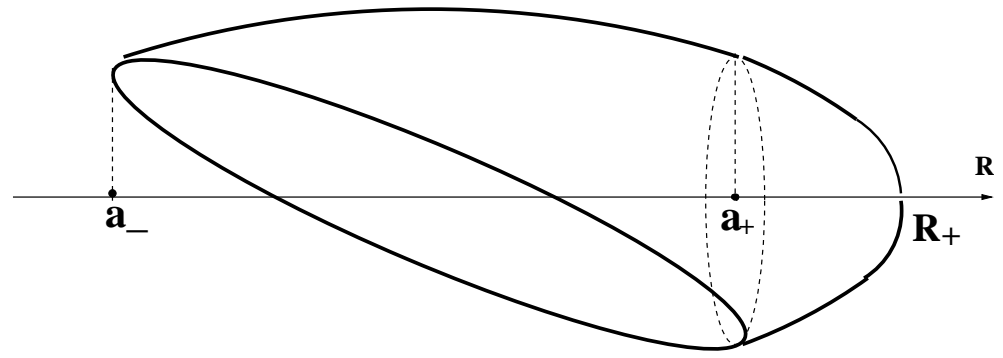

Figure 3: Truncation of the 5D Schwarzschild-de Sitter spacetime excluding from the bulk the Schwarzschild horizon point $R_{-}$.

Therefore, a natural selection criterion for the regularity at the deSitter horizon $R_{+}$becomes just one equation relating $\Delta T_{+}$and $\Delta T$

$$
2 k \int_{\tau_{-}}^{\tau_{+}} d \tau \frac{\sqrt{f(a)-a^{\prime 2}}}{f(a)}=\frac{\pi \sqrt{2}}{H}\left(\frac{1+\sqrt{1-4 R_{S}^{2} H^{2}}}{1-4 R_{S}^{2} H^{2}}\right)^{1 / 2} .
$$

This is an equation on $\mathcal{C}=R_{S}^{2}$ in terms of $H^{2}$ and $B$ analogous to the bootstrap equation (10) on the $4 \mathrm{D}$ CFT side of this problem. It does not however coincide with (10), so that the suggested DGP/CFT duality does not incorporate a 
complete equivalence of $4 \mathrm{D}$ and $5 \mathrm{D}$ pictures for the full admissible range of $\Lambda=3 H^{2}$ on the CFT side. If we insist on the full correspondence of these descriptions, this will lead to the additional restriction of the $H^{2}$-range - the selection of a finite set of instantons with isolated values of the cosmological constant. Below we derive those values for static instantons corresponding to static Einstein universes and for garlands generated by a conformal scalar field.

\section{Conical singularity in the bulk vs static Ein- stein universes and garlands}

For a particular value of the amount of radiation constant - at the hyperbolic boundary of the instanton domain in Fig 1

$$
\mathcal{C}=\frac{1-2 B H^{2}}{4 H^{2}}
$$

the effective equations (9)-(10) have a one-parameter set of solutions corresponding to static Einstein universes with a constant $a(\tau)=a_{+}=a_{-}=1 / H \sqrt{2}$. Since for constant $a(\tau)$ the solution can be considered periodic with an arbitrary period $\eta=\oint d \tau / a=\sqrt{2} H \Delta \tau$, the bootstrap equation (10) does not impose any additional restriction on the value of $H$ and only determines $H$ in terms of $\eta$ (or other way around $-\eta$ in terms of $H$ )

$$
\begin{aligned}
& 2 B H^{2}=\frac{1}{1+r(\eta)}, \\
& r(\eta)=\frac{2}{B m_{P}^{2}} \frac{d F(\eta)}{d \eta}=\frac{E_{\mathrm{rad}}(\eta)}{E_{\mathrm{vac}}}
\end{aligned}
$$

where $r(\eta)$ is in fact a ratio of the radiation energy to the vacuum Casimir energy 6

Static solutions exist for any $H$ in the range $0 \leq H^{2} \leq 1 / 2 B$. In particular, for a small $H \rightarrow 0$ they have a period $\eta \rightarrow 0$ which in view of the asymptotic behavior $d F / d \eta \simeq \pi^{4} / 15 \eta^{4}$ (in the case of a scalar field) reads

$$
\eta \simeq \pi \sqrt{\frac{H}{m_{P}}}\left(\frac{4}{15}\right)^{1 / 4} .
$$

This generates the on-shell value of the effective action (which is given on the static solutions by the Legendre transform of $F(\eta)$ [4]

$$
\Gamma_{0}=F-\eta \frac{d F}{d \eta} \simeq-\frac{\pi}{3}\left(\frac{4}{15}\right)^{1 / 4}\left(\frac{m_{P}}{H}\right)^{3 / 2} \rightarrow-\infty, \quad H \rightarrow 0 .
$$

\footnotetext{
${ }^{6}$ Since $r(\eta)$ is a monotonically decreasing function of $\eta$, this relation determines a one-toone correspondence between $H$ and $\eta$.
} 
Therefore these instantons weighted by $\exp \left(-\Gamma_{0}\right)$ are strongly dominated by the $H \rightarrow 0$ limit - the analogue of the infrared catastrophe with the HartleHawking no-boundary wavefunction treated in the tree-level approximation 7 .

This singularity is not dangerous for physical implications in early Universe, because these static Euclidean solutions are stable and do not give rise to Lorentzian expanding universes modeling initial conditions for inflationary cosmology 8. They represent thermodynamic equilibrium ensembles with the temperature $T=1 / \Delta \tau \simeq(15)^{1 / 4} \sqrt{m_{P} H} / \pi \rightarrow 0$. The same is true for conformal fields of other spins - they generate the same power law behavior for the cosmological parameters, differing only by spin-dependent coefficients. It is interesting, that the dual 5D picture of the DGP/CFT correspondence provides a strong truncation of this family of solutions by selecting just one member from the range $0 \leq H^{2} \leq 1 / 2 B$.

This truncation follows from requiring the absence of a conical singularity at the de Sitter horizon of the 5D bulk (50). Indeed, in the static case (51) both sides of this equation simplify, because $a^{\prime}=0, f(a)=B H^{2}$ and $1-4 R_{S}^{2} H^{2}=$ $2 B H^{2}$, and it takes the form

$$
\eta_{\text {cone }}=\pi \sqrt{2\left(1+\sqrt{2 B H^{2}}\right)}
$$

The substitution of this expression to (52) gives a closed equation for the numerical value of $2 \mathrm{BH}^{2}$. This equation always has a solution between zero and one. Note that the period for the coneless case has a very limited range $\pi \sqrt{2} \leq \eta \leq 2 \pi$ in which the evaluation of $F(\eta)$, (4), with a big accuracy is given by the contribution of several lowest oscillator levels $\omega$. The numerical solution of this equation does not present any difficulty, and it gives, for a pure scalar field with $B m_{P}^{2}=1 / 240$

$$
2 B H^{2} \simeq 0.3612, r \simeq 1.7688,
$$

(the corresponding thermal sum of (10) runs over the eigenfrequencies on a unit 3 -sphere $\omega_{n}=n$ with weights $\sum_{\omega} \equiv \sum_{n \geq 1} n^{2}$ ).

For a pure 2-component Weyl spinor field with $B m_{P}^{2}=11 / 480, \omega_{n}=n+1 / 2$ and $\sum_{\omega} \equiv \sum_{n \geq 1} n(n+1)$ we have

$$
2 B H^{2} \simeq 0.9858, \quad r \simeq 0.0144,
$$

and for a pure vector field with $B m_{P}^{2}=31 / 120, \omega_{n}^{2}=2\left(n^{2}-2\right)$ and $\sum_{\omega} \equiv$ $2 \sum_{n \geq 2}\left(n^{2}-1\right)$

$$
2 B H^{2} \simeq 0.9998, \quad r \simeq 0.00016 \ll 1 .
$$

Interestingly, for spinors and vectors the cone free instantons are very close to the upper bound of the range (12) - the new quantum gravity scale $H^{2}=1 / 2 B$.

\footnotetext{
${ }^{7}$ Note, however, that the singularity of $\exp \left(-\Gamma_{0}\right)$ is weaker here because for the HartleHawking prescription the tree-level action $-\Gamma_{0} \sim\left(m_{P} / H\right)^{2}$ in contrast to $\left(m_{P} / H\right)^{3 / 2}$ in our case.

${ }^{8}$ For this reason these solutions were not considered in [4].
} 
When the CFT is composed of many fields of one spin, these values do not depend on their number $N$ (because $N$ cancels out in the ratio (53). In the case of the mixture of spins, they depend on the partial weights of these spins in the full system. Then the resulting value of $2 \mathrm{BH}^{2}$ lies somewhere between (57) and (59).

Thus, only one member of the static Einstein family with $\Lambda \neq 0$ remains in a dual DGP/CFT description, which again eliminates the infrared catastrophe of $\Lambda \rightarrow 0$ (even though this catastrophe for this family is irrelevant from the viewpoint of early cosmology). Application of the same criterion of dual description to garland families by the combination of Eqs.(50) and (10) also restricts them to a finite set (intersection of the one-parameter set of solutions of (50) with one-parameter sets of garlands). In view of the irreducible integral nature of these equations this can hardly be done analytically. Numerical calculations show that for the scalar field there is a single member of the $k=2$ garland family with the following value of the Hubble constant and the amount of radiation constant

$$
H_{\text {garland }}^{2} \simeq 56.5 m_{P}^{2}, \quad \mathcal{C}=0.0018 m_{P}^{-2} .
$$

In fact this instanton is rather close to the static instanton of (57).

For other spins and their mixtures we do not have concrete results, but it is expected that a dual description also exists for a finite set of garlands with isolated values of the cosmological constant. This problem will be analyzed elsewhere. In particular, it is anticipated that, when vector and spinor contributions dominate in the particle phenomenology, the DGP/CFT duality will enforce the values of the cosmological and amount of radiation constants close to (58)-(59), that is very close to the upper boundary of the range (12).

This duality is very much equivalent to the AdS/CFT correspondence picture not only from the viewpoint of classical-to-quantum correspondence, but also from the viewpoint of energy scales of the problems. Indeed, on the CFT cosmology side the limit $2 B H^{2} \simeq 1$ corresponds to the uppermost possible value of $\Lambda$. With the number of conformal fields $N \gg 1$ the corresponding $\Lambda / 3 \simeq 1 / 2 B \sim m_{P}^{2} / N$ is small, but it still turns out to be in a strong coupling limit - at the cutoff generated in gravitational physics by a large number of quantum species [2, 3]. On the $5 \mathrm{D}$ side, however, we have for large $N$ large semiclassical black holes. This follows from the fact that for large $B \sim N$ the black hole horizon in the vicinity of the family of solutions (51) can be estimated as

$$
R_{S}^{2}=\mathcal{C} \simeq \frac{B}{2} r \sim \frac{N}{m_{P}^{2}} r \gg m_{P}^{-2},
$$

because the radiation to vacuum energy ratio (53) is a quantity essentially independent of the total $N$. Thus, this is a nearly classical black hole provided the number of species is larger than $1 / r$. Moreover the dynamics of the brane cosmology takes place at $a_{ \pm}^{2}=1 / 2 H^{2} \simeq B \sim N / m_{P}^{2}$ far away from the $\mathrm{BH}$ horizon, where quantum effects of the bulk background are even weaker. 


\section{Towards background independent duality}

The duality between the 4D CFT setting and 5D braneworld setup holds in a cosmological sector, with degrees of freedom which are spatially homogeneous collective variables. The duality between both models is far from being obvious when those degrees of freedom are not restricted to be homogeneous, as is the case for the background cosmology, but are also free to contain spatially inhomogeneous modes. This can be checked only by comparing relevant sets of correlation functions considered within perturbation theory on this background. However, the duality construction of the above type can be considered as a step towards background independent classical-to-quantum correspondence between the theories in neighboring spacetime dimensions [7. Our case goes beyond a conventional AdS/CFT correspondence, where the bulk Schwarzschild-AdS background and its asymptotically remote boundary are in fact non-dynamical (being a trivial static solution of 10D SUGRA equations parameterized by fixed coupling constants). Here the $4 \mathrm{D}$ thermal brane is located at a finite variable distance from the $\mathrm{BH}$ and undergoes a nontrivial evolution in the bulk background. The latter is static but involves the $\mathrm{BH}$ mass (and the relevant amount of radiation on the brane) which is not a free input but rather a quantity fixed by nontrivial consistency conditions.

Moreover, this model contains the indication that this duality can be extended further, beyond any assumptions on spacetime symmetries of the underlying background. As we will see, a part of the full nonlinear equations in both settings of this model coincide irrespective of the particular symmetry ansatz for the physical configurations. To show this, consider the Einstein equations with Israel boundary (junction) conditions (25)-(27). With the DGP scale parameter (17) the junction conditions can be rewritten as

$$
K_{\mu \nu}=-r_{c}\left(R_{\mu \nu}-\frac{1}{6} g_{\mu \nu} R\right)
$$

and the gravitational constraint in the bulk - the normal-normal projection of (25),

$$
\begin{aligned}
-2 n^{A} n^{B}\left(R_{A B}^{(5)}-\frac{1}{2} G_{A B}\right. & \left.R^{(5)}+\Lambda_{5} G_{A B}\right) \\
= & R^{(4)}+K_{\mu \nu}^{2}-K^{2}-2 \Lambda_{5}=0,
\end{aligned}
$$

takes the form of the following equation entirely in terms of $4 \mathrm{D}$ brane quantities

$$
R^{(4)}+r_{c}^{2}\left(R_{\mu \nu}^{2}-\frac{1}{3} R^{2}\right)-2 \Lambda_{5}=0,
$$

because the quadratic form in the extrinsic curvature reads as $K_{\mu \nu}^{2}-K^{2}=$ $r_{c}^{2}\left(R_{\mu \nu}^{2}-R^{2} / 3\right)$ - a structure related by a total derivative term to the square of the Weyl tensor. Thus, unexpected conformal properties start popping out in this seemingly unrelated to conformal invariance braneworld setup. The treelevel equation (64) is exact and totally independent of any background ansatz restrictions. 
Let us now consider the quantum effective equations on the 4D CFT side. Without any symmetry assumptions and selection of collective variables the full effective action in the theory (in the $1 / N$-approximation) can be represented as a sum of Einstein term, the conformal anomaly action $\Gamma_{A}$ and conformally invariant part including the action for thermal radiation and vacuum (Casimir) energy $\Gamma_{T, C}$

$$
\Gamma=-\frac{1}{16 \pi G_{4}} \int d^{4} x g^{1 / 2}\left(R^{(4)}-2 \Lambda_{4}\right)+\Gamma_{A}+\Gamma_{T, C} .
$$

Now consider the conformal variation of this action or the trace part of effective Einstein equations. We will assume that, as before, the number of degrees of freedom preserving renormalization has been done, so that the conformal variation of the anomalous action has the form (77) with the coefficient of the $\square R$ term $\alpha=0$. With a known equation for the Gauss-Bonnet invariant $E=R_{\mu \nu \alpha \gamma}^{2}-4 R_{\mu \nu}^{2}+R^{2}$ in terms of the Weyl tensor

$$
E=C_{\mu \nu \alpha \beta}^{2}-2\left(R_{\mu \nu}^{2}-\frac{1}{3} R^{2}\right)
$$

this anomalous variation reads

$$
g_{\mu \nu} \frac{\delta \Gamma_{A}}{\delta g_{\mu \nu}}=\frac{1}{64 \pi^{2}} g^{1 / 2}\left[-2 \beta\left(R_{\mu \nu}^{2}-\frac{1}{3} R^{2}\right)+(\beta+\gamma) C_{\mu \nu \alpha \beta}^{2}\right] .
$$

Therefore, in view of the local conformal invariance of $\Gamma_{T, C}$ the full trace effective equation $g_{\mu \nu} \delta \Gamma / \delta g_{\mu \nu}=0$ takes the form

$$
R+\frac{\beta G_{4}}{2 \pi}\left(R_{\mu \nu}^{2}-\frac{1}{3} R^{2}\right)-4 \Lambda_{4}-\frac{\beta+\gamma}{4 \pi} G_{4} C_{\mu \nu \alpha \beta}^{2}=0 .
$$

Under the known indentifications of the 4D and 5D parameters (19)-(20), the first three terms here exactly reproduce the equation (64) on a 5D braneworld side - the tree-level generalized DGP model. For a cosmological setting with a conformally flat FRW metric the last term is vanishing, but for a generic field configurations it is nonzero, and these $5 \mathrm{D}$ and $4 \mathrm{D}$ pictures are generally not dual. However, salvation might come from the supersymmetry requirement. It is a well-known fact that in the superconformal field theory - the $D=4$ $S U(\mathbb{N}) S Y M$ with an extended $\mathcal{N}=4$ supersymmetry [12, 22, which is a typical object of AdS/CFT correspondence with the particle contents $\left(N_{0}, N_{1 / 2}, N_{1}\right)=$ $\left(6 \mathbb{N}^{2}, 4 \mathbb{N}^{2}, \mathbb{N}^{2}\right)$, cf. Eq.(8), - the coefficients of the conformal anomaly satisfy the relation

$$
\gamma+\beta=0 .
$$

This renders equations (64) and (68) completely equivalent for a generic metric configuration. In particular, the particle model with the same phenomenology 9 when applied in our CFT cosmology will provide a hierarchy of classical-toquantum coupling scale limits (61) with $N \sim \mathbb{N}^{2} \gg 1$.

\footnotetext{
${ }^{9}$ One should bear in mind that this will not be literally the SCFT of the above type, because of a positive cosmological constant.
} 
One can find for this particle content the static instanton (on the hyperbolic curve of Fig (1) satisfying the selection criteria on both 4D and 5D sides. According to the previous section, it represents a numerical solution of Eq.(52) with the conformal time (56) for the above set of spins with the relevant value of the total $B m_{P}^{2}=\left(6 B_{0}+4 B_{1 / 2}+B_{1}\right) m_{P}^{2} \mathbb{N}^{2}=3 \mathbb{N}^{2} / 8$ and the relevant thermal scalar, spinor and vector sums weighted in $d F(\eta) / d \eta$ by the numbers of particles $N_{s}$, cf. Eq.(10). It turns out that this static instanton has the parameters

$$
2 B H^{2} \simeq 0.937, r \simeq 0.0673,
$$

which again nearly saturate the cutoff bound $H^{2}=1 / 2 B$. Apparently, the garland instanton satisfying the same criteria of a complete duality is also very close to this point, which supports the conclusions of Sect.5 on the weak coupling to strong coupling correspondence of relevant $5 \mathrm{D}$ and $4 \mathrm{D}$ pictures.

\section{The case of a negative cosmological constant}

The previous section gives a strong motivation for the introduction of supersymmetry in the particle phenomenology of the model, which however requires negative values of the bulk and brane cosmological constants in (20). For this reason let us consider the case of

$$
\Lambda_{4} \equiv-3 H^{2}<0 .
$$

We will see that this results in the flip of the sign of the effective cosmological constant, which makes this choice useful also for the use in inflation theory.

With this choice the formal turning points (11) go over into

$$
a_{ \pm}^{2}=\frac{ \pm \sqrt{1+2 B H^{2}+4 \mathcal{C} H^{2}}-1}{2 H^{2}},
$$

and in view of $a_{-}^{2}<0$ the Euclidean evolution of the scale factor can only be in the range $0 \leq a(\tau) \leq a_{+}$, which corresponds to the formation of the Hartle-Hawking vacuum instanton with the $S^{4}$ topology - degenerate case of a torus ripped at the vanishing value of the scale factor. The Euclidean evolution domain $a(\tau) \geq a_{+}$is of no interest, because it is not periodic and of no relevance to the statistical sum of the model.

With $a=0$ at the boundary of the evolution domain the conformal time diverges to infinity, $\eta \rightarrow \infty$, so that $d F(\eta) / d \eta \rightarrow 0$, and $\mathcal{C}=0$ from (10). Then the effective Friedmann equation (9) reduces to

$$
\begin{aligned}
& a^{\prime 2}=1-a^{2} \frac{1 \pm \sqrt{1+2 B H^{2}}}{B}=1-H_{\mathrm{eff}}^{2} a^{2}, \\
& H_{\mathrm{eff}}^{2}=\frac{1+\sqrt{1+2 B H^{2}}}{B},
\end{aligned}
$$

where the plus sign of the square root in the expression for $a^{\prime 2}$ is chosen to have a necessary turning point at $a_{+}=1 / H_{\text {eff }}$ - the equatorial section of 
the spherical Hartle-Hawking instanton mentioned above. The solution of (73) is an obvious Euclidean de Sitter metric with a positive effective cosmological constant $\Lambda_{\text {eff }}=3 H_{\text {eff }}^{2}$ (cf. Eq.(13) for the Hartle-Hawking instanton in the case of a positive primordial $\Lambda=3 H^{2}$ ). When rewritten in terms of the original negative cosmological constant (71) this effective one explicitly features the sign flip

$$
\Lambda_{\mathrm{eff}}=-\frac{2 \Lambda_{4}}{\sqrt{1-2 B \Lambda_{4} / 3}-1}>0 .
$$

This nonlinear transition from the negative primordial cosmological constant to the positive effective one is a genuine quantum effect of the conformal anomaly. This effect, for example, makes unnecessary the KKLT uplifting [25] of the negative vacuum energy - here the primordial cosmological constant can be negative and consistent with supersymmetry and still can generate a positive effective $\Lambda_{\text {eff }}$ needed for inflation.

Unfortunately, there is an obstacle to the realization of this elegant mechanism. If we believe in the microcanonical initial conditions of the above type, then the contribution of the Hartle-Hawking instanton should be weighted by the exponentiated negative of the Euclidean effective action. But, as mentioned above, the latter diverges to $+\infty$ [4, 5] by virtue of the conformal anomaly part and suppresses to zero this contribution of a primordial $\Lambda_{4}<0$. There is a hope to overcome this difficulty by using the tunneling prescription [26, 27] alternative to the no-boundary construction of the above type. As suggested in [28] the tunneling state can be incorporated into the microcanonical path integral formulation in the form of another saddle point of (2) with the negative value of the lapse function $N=-1$. In the main it leads to the reversal of the sign of the semiclassical exponential in the distribution function of the quantum ensemble. But such a tunneling state was calculated in [28] only for a system with non-conformal quantum fields with heavy masses, admitting a local gradient expansion of the effective action. The inclusion of massless conformal fields into this construction encounters certain difficulties and is currently under study.

\section{Conclusions}

Thus we have constructed a 5D braneworld setup dual to the microcanonical state of a $4 \mathrm{D}$ spatially closed cosmology driven by the conformal field theory with a large number of quantum fields. The importance of this model lies in the fact that it provides initial conditions for inflationary cosmology in a limited range of the effective cosmological Hubble factor, which at late stages of expansion can also have a stage of the cosmological acceleration by the big boost scenario of Ref. 6]. The dual 5D picture is represented by the generalized DGP model with no matter on the brane, but with a positive primordial cosmological constant in the bulk and a black hole imitating the radiation of conformal fields on the cosmological 4D CFT side. For a large number of quantum CFT species $N$ the DGP/CFT duality holds for distinguished values of the cosmological constant, 
which correspond to the classical weak coupling regime of the 5D Schwarzschildde Sitter bulk and a strong coupling regime of the 4D CFT at the natural cutoff induced by large $N$.

This duality is fully proven for global cosmological degrees of freedom of the system, and its extension to the sector of spatially inhomogeneous modes and their perturbative correlators still has to be done. However, there is an indication that this can be done even at the level of the full nonlinear equations, because a trace part of effective Einstein equations exactly coincides on both sides of the duality relation for a generic metric configuration unrestricted by any Killing symmetry considerations. This only imposes constraints on particle phenomenology provided, in particular, by superconformal symmetry of the theory. This suggests a new type of background independent classical-to-quantum correspondence. Finally, we proposed a mechanism for flipping the sign of the effective cosmological constant in the CFT driven cosmology, which can reconcile the negative, compatible with supersymmetry, value of the primordial cosmological constant with inflationary cosmology.

\section{Acknowledgements}

A.B. is grateful for hospitality of the Laboratory APC in Paris (CNRS, Paris VII University, CEA, Paris Observatory) and the Laboratory MPT CNRS-UMR 6083 of the University of Tours, where a major part of this work has been done. His work was also supported by the Russian Foundation for Basic Research under the grant No 08-01-00737 and the grant LSS-1615.2008.2. A.K. was supported by the RFBR grant 08-02-00725 and the grant LSS-4899.2008.2. A.B. wishes to thank G.Dvali, P.Mazur, E.Mottola, M.Shaposhnikov, S.Solodukhin, A.Starobinsky and R.Woodard for discussions.

\section{References}

[1] Maldacena J, 1998 Adv. Theor. Math. Phys. 2 231; 1999 Int. J. Theor.Phys. 38 1113; Witten E, 1998 Adv. Theor. Math. Phys. 2 253; Gubser S S, Klebanov I R and Polyakov A M, 1998 Phys. Lett. B 428105.

[2] Dvali G, Black Holes and Large $N$ Species Solution to the Hierarchy Problem, e-Print: arXiv:0706.2050 [hep-th].

[3] Dvali G and Redi M, Phys. Rev. D77 045027, 2008, e-Print: arXiv:0710.4344 [hep-th]; Dvali G and Solodukhin S N, Black Hole Entropy and Gravity Cutoff, CERN-PH-TH-2008-141, Jun 2008. 11pp. ePrint: arXiv:0806.3976 [hep-th].

[4] Barvinsky A O and Kamenshchik A Yu, JCAP09 (2006) 014; Phys. Rev. D 74121502 .

[5] Barvinsky A O, 2007 Phys. Rev. Lett. 99071301. 
[6] Barvinsky A O, Deffayet C and Kamenshchik A Yu, JCAP 05 (2008) 020, arXiv:0801.2063.

[7] Dvali G and Gomez C, 2009 Phys. Lett. B 674 303, e-print: arXiv:0812.1940 [hep-th]; Strong Coupling Holography, e-print: arXiv:0907.3237 [hep-th].

[8] Riegert R J, 1984 Phys. Lett. B 134 56; Mazur P O and Mottola E, 2001 Phys. Rev. D 64 104022; Fradkin E S and Tseytlin A A, 1984 Phys. Lett. B 134187.

[9] Starobinsky A A, 1980 Phys. Lett. B 9199.

[10] Hartle J B and Hawking S W, 1983 Phys.Rev. D 282960.

[11] Hawking S W, Hertog T and Reall H S, 2000 Phys.Rev. D 62043501.

[12] Duff M J, 1994 Class. Quant. Grav 11 1387; Duff M J and Liu J T, 2000 Phys. Rev. Lett. 852052.

[13] Brown L S and Cassidy J P, 1977 Phys. Rev. D 16 1712; Antoniadis I, Mazur P O and Mottola E, 1997 Phys. Rev. D 55 4770, e-print: arXiv:9509169[hep-th].

[14] J.S.Dowker and R.Critchley, J. Phys. A 9, 535 (1976); S.G.Mamaev, V.M.Mostepanenko and A.A.Starobinsky, Sov. Phys. JETP, 43, 823 (1976); L.H.Ford, Phys. Rev. D 14, 3304 (1976); P.Candelas and J.S.Dowker, Phys. Rev. D 19, 2902 (1979).

[15] Dvali G R, Gabadadze G and Porrati M, 2000 Phys. Lett. B 485208.

[16] Deffayet C, 2001 Phys. Lett. B 502199.

[17] Binetruy P, Deffayet C, Ellwanger U and Langlois D, 2000 Phys. Lett. B 477285.

[18] Shiromizu T, Maeda K i and Sasaki M, 2000 Phys. Rev. D 62024012.

[19] Kraus P, 1999 JHEP 9912011.

[20] Deffayet C, Dvali G and Gabadadze G, 2002 Phys. Rev. D 65044023.

[21] Luty M A, Porrati M and Rattazzi R, 2003 JHEP 0309 029; Nicolis A and Rattazzi R, 2004 JHEP 0406 059; Dvali G, 2006 New J. Phys. 8 326; Koyama K, 2005 Phys. Rev. D 72123511 Gorbunov D, Koyama K and Sibiryakov S, 2006 Phys. Rev. D 73 044016; Charmousis C, Gregory R, Kaloper N and Padilla A, 2006 JHEP 0610 066; Deffayet C, Gabadadze G and Iglesias A, 2006 J. Cosmol. Astropart. Phys. JCAP08(2006)012; Izumi K, Koyama K, Pujolas O and Tanaka T, 2007 Phys. Rev. D 76104041 Gregory R, Kaloper N, Myers R C and Padilla A, JHEP 0710069.

[22] Hong Liu and Tseytlin A A, 1998 Nucl.Phys. B 53388. 
[23] G.W.Gibbons and S.W.Hawking, Phys. Rev. D15 (1977) 2738.

[24] S.W.Hawking and D.Page, Commun. Math. Phys. 87 (1983) 577.

[25] S.Kachru, R.Kallosh, A.Linde and S.P.Trivedi, Phys.Rev. D 68, 046005 (2003); S.Kachru, R.Kallosh, A.Linde, J.Maldacena, L.McAllister and S.P.Trivedi, JCAP 10, 013 (2003).

[26] A. D. Linde, JETP 60 (1984) 211; Lett. Nuovo Cim. 39 (1984) 401; V. A. Rubakov, JETP Lett. 39 (1984) 107; Ya. B. Zeldovich and A. A. Starobinsky, Sov. Astron. Lett. 10 (1984) 135.

[27] A. Vilenkin, Phys. Rev. D 30 (1984) 509; Phys. Rev. D 50 (1994) 2581.

[28] A.O. Barvinsky, A.Yu. Kamenshchik, C. Kiefer and C. Steinwachs, Tunneling cosmological state revisited: Origin of inflation with a non-minimally coupled SM Higgs inflaton, e-print: arXiv:0911.1408 [hep-th]. 